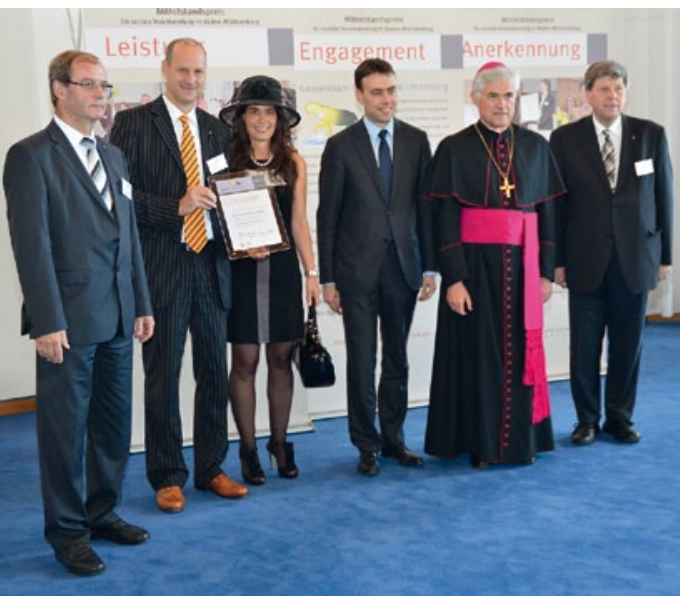

Am 26. Juni wurden mittelständische Unternehmen in Stuttgart mit dem „Mittelstandspreis für soziale Verantwortung in Baden-Württemberg 2012“ ausgezeichnet. $\mathrm{Zu}$ den nominierten Unternehmen,
V.I.n.r.: Prälat Wolfgang Tripp, Martin Dürrstein, Esther Dürrstein, Dr. Nils Schmid MdL, Weihbischof Dr. Bernd Uhl, Monsignore Bernhard Appel.

die aus 215 Bewerbungen ausgewählt wurden, gehört auch die DÜRR DENTAL AG aus Bietigheim-Bissingen. „Soziale Verantwortung ist uns ein besonderes Anliegen. Wir engagieren uns vornehmlich im Bereich Gesundheit. International für Mercy Ships und als Bekenntnis zur Region bildet die Förderung ansässiger Sportvereine ein Schwerpunkt“, erläuterte Vorstandsvorsitzender Martin Dürrstein nach der Urkundenverleihung. Der Bietigheimer Medizintechnik-Spezialist engagiert sich seit 10 Jahren vor allem für die Hilfsorganisation Mercy Ships, die in Afrika das größte private Hospitalschiff „Africa Mercy“ betreibt - ein Schiff mit einer Besatzung aus Chirurgen, Krankenschwestern, Bauleuten, Landwirtschaftsexperten und vielen weiteren Helfern. 400 Ehrenamtliche aus der ganzen Welt begleiten das Hospitalschiff.

Das Familienunternehmen stattet die Hilfsorganisation mit Produkten für seine mobilen Zahnkliniken aus. Darüber hinaus wurde bereits 2-mal die Aktion „Ein neues Lachen für Afrika“ durchgeführt. Um die zahngesundheitliche Situation in Westafrika ein Stück weit zu verbessern, hat das Unternehmen für jede bis Ende 2011 verkaufte Druckluft- und Absauganlage eine Zahnbehandlung gespendet. Um weitere Zahnärzte für den Einsatz auf der Africa Mercy zu motivieren, wurde ein Infoabend durchgeführt.

Nach einer Pressemitteilung der DÜRR DENTAL AG, Bietigheim-Bissingen Internet: www.duerr.de

\title{
50 Jahre dentale Innovationen am Standort Bensheim
}

2012 ist ein 3-faches Jubiläumsjahr für Sirona: Neben dem runden 50. Standortjubiläum in Bensheim blickt das Dentalunternehmen auf eine 135 Jahre alte Firmengeschichte und auf 15 Jahre seit Gründung der Sirona Dental Systems GmbH zurück. 1962 bauten die Siemens Reiniger Werke aus Erlangen einen neuen Standort in Südhessen auf und legten damit den Grundstein für eine der heute modernsten und größten Dentalfabriken weltweit. Das Unternehmen präsentierte sich zum 50. Standortjubiläum in Bensheim mit vielen dentalen Innovationen sowie mit neuen Gebäuden in herausragender Gestaltung. Das 2011 in Betrieb genommene Sirona Innovationszentrum in modernstem Design beherbergt mittlerweile mehr als 240 Ingenieure und Mitarbeiter der Entwicklung

Das Jahr 2012 ist für Sirona gleich ein mehrfaches Jubiläumsjahr. So reichen die Wurzeln des dentalen Weltmarktführers bis in das Jahr 1877 zurück. Vor genau 135 Jahren fertigte der Universitätsmechaniker Erwin Moritz Reiniger, einer der Gründerväter des Unternehmens, erste elektromedizinische und physikalische Apparate. Aus seinem Unternehmen Rei-

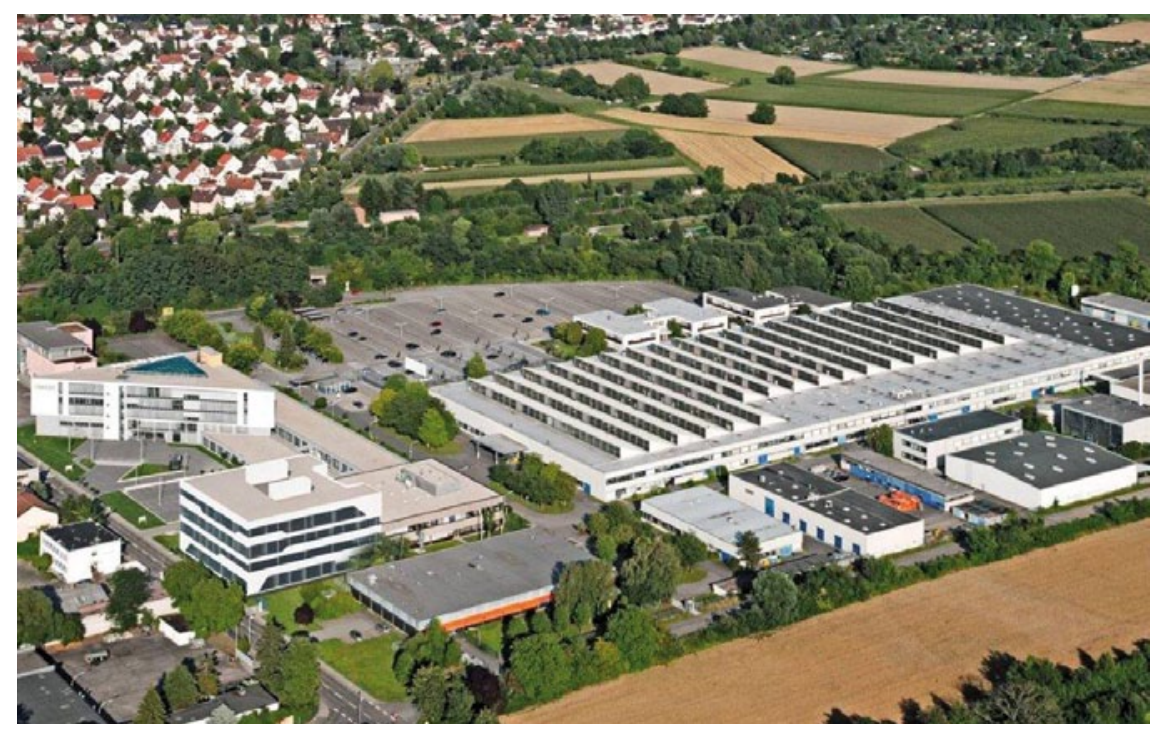

Der Sirona-Standort Bensheim heute.

niger Gebbert \& Schall entstanden die Siemens Reiniger Werke, die später dann in der Siemens AG aufgingen. Ein weiteres denkwürdiges Jahr war 1997, als Siemens sich von der Dentalsparte trennte, die dann zur Sirona Dental Systems GmbH wurde.

Mit dem Umzug der Vertriebs- und Entwicklungsabteilungen von Erlangen nach
Bensheim im Jahr 1969 war das gesamte Geschäftsgebiet „Siemens Dental“ am neuen Standort vertreten. Heute ist das Unternehmen Technologie- und Weltmarktführer der Dentalindustrie mit 24 Standorten und einem globalen Vertriebs- und Servicenetz.

Nach einer Pressemitteilung der Sirona Dental Systems GmbH, Bensheim Internet: www.sirona.de 


\section{André Schroeder-Forschungspreis 2013}

Alle Wissenschaftler, die über neue Forschungserkenntnisse auf dem Gebiet der dentalen Implantologie verfügen, können sich ab sofort für den André SchroederForschungspreis 2013 bewerben. Die Bewerbungsfrist für den jährlich vom Internationalen Team für Implantologie (ITI) vergebenen Preis endet am 15. Oktober 2012. Der Gewinner erhält ein Preisgeld in Höhe von 20000 Schweizer Franken. Außerdem wird er zum ITI-Kongress Nordamerika nach Chicago (4.-6. April 2013) eingeladen, an dem der André SchroederForschungspreis 2013 verliehen wird.

Der André Schroeder-Forschungspreis zählt zu den renommiertesten Auszeichnungen in der dentalen Implantologie. Er wird seit 20 Jahren an unabhängige Wissenschaftler vergeben, um neue wissenschaftliche Erkenntnisse in zahnmedizinischer Forschung zu fördern. Das ITI verleiht den Preis zu Ehren von Prof. André Schroeder (1918-2004), der die moderne Zahnheilkunde maßgeblich beeinflusst hat und
Gründungspräsident des ITI war. Die Preisträger werden vom ITI-Forschungskomitee ermittelt, das sich aus international anerkannten Experten zusammensetzt.

Das Bewerbungsformular für den André Schroeder-Forschungspreis 2013 sowie weitere Informationen $\mathrm{zu}$ den Teilnahmebedingungen sind auf der Webseite des ITI verfügbar: www.iti.org.

Nach einer Pressemitteilung des ITI International Team for Implantology, ITI Center

\section{Special Olympics \\ Strahlende Athleten}

Begeisterung bei den Special Olympics Ende Mai in München: 5000 Sportler mit geistiger Behinderung aus ganz Deutschland maßen sich in 19 Disziplinen und wurden dabei auch zahngesundheitlich top betreut. Hierfür sorgte das Gesundheitsprogramm Special Smiles Deutschland, das von der Wrigley GmbH unterstützt wird. Es ist das größte Engagement innerhalb der unternehmerischen Sozialverantwortung des Unternehmens. Rund ein Viertel der Belegschaft von Wrigley Deutschland war in München vor Ort. Zahnärzte und Helfer zeigten den Athleten, wie sich Zähne auch mit Handicap erfolgreich putzen lassen. Insgesamt 731 Athleten ließen sich nach einem spezifischen Screening-Programm untersuchen.
Das Ergebnis: 426 Athleten (58\%) wiesen Gingivitis auf, und 150 Athleten (21\%) mussten dringend zahnärztlich behandelt werden. „Gerade für Menschen mit geistigen Behinderungen stellt das Zähneputzen eine große Herausforderung dar. Denn hier ist feinmotorisches Geschick notwendig, das diese Menschen oft nicht so schnell erlernen wie Menschen ohne Behinderung“, erklärte die Zahnärztin Dr. Imke Kaschke, Leiterin Healthy Athletes. Doch so wie beim Sport gaben die Athleten in München auch bei der Mundpflege ihr Bestes. Sportler und Helfer hatten ihren Spaß daran - getreu dem Motto der Spiele „Gemeinsam sind wir stark“. Wrigley engagiert sich seit über 20 Jahren für gesunde Zähne, unter

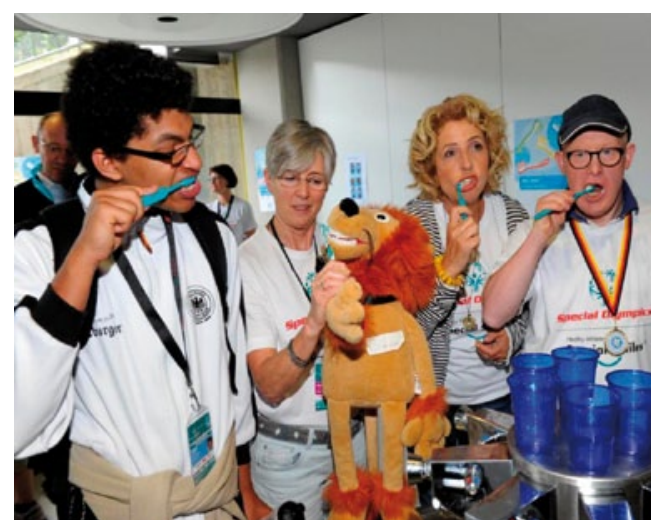

anderem mit seinem Angebot zuckerfreier Zahnpflegekaugummis und Pastillen zur Mundpflege.

Nach einer Pressemitteilung des Wrigley Orals Health Program Internet: www.wrigley-dental.de

\section{Kostenfreie Checkliste für mehr}

\section{Internet-Kompetenz}

Neue Bezugspreise ab 1. Januar 2013

Laut einer Studie der Stiftung Gesundheit sind Ärzte offen für Onlinemarketing und planen Budgets dafür ein. Doch welche Investition lohnt sich wirklich und wie lässt sich der Erfolg kontrollieren? Die Gesellschaft für Zahngesundheit, Funktion und Ästhetik (GZFA) bietet nun Zahnärzten und Zahntechnikern eine Checkliste mit Leitfragen. Sie steht zum kostenfreien Herunterladen auf dem Portal www.gzfa.de bereit. „Damit lässt sich der eigene Status festmachen und der Blick für zukunftsträchtige Optionen öffnen“, so Franz Weiß, Geschäftsführer der GZFA. Die GZFA steht für ein bundesweites zahnärztliches Netzwerk. Sie betreibt seit 2004 erfolgreich ein Portal zum Thema Zahngesundheit sowie mehrere themenspezifische Onlineverzeichnisse.

Nach einer Pressemitteilung der Gesellschaft für Zahngesundheit, Funktion und Ästhetik mbH (GZFA), München

Internet: www.gzfa.de
Der Verlag bemüht sich, die Bezugspreise für Zeitschriften stabil zu halten. Leider müssen wir ab dem Jahrgang 2013 die Preise der ZWR etwas anheben, um einen Teil der gestiegenen Herstellungs- und Vertriebskosten abzudecken. Ab Januar 2013 berechnen wir für das Einzelheft 34 ,- $€$, für das Jahresabo $226,-€$, Studenten erhalten ein ermäßigtes Jahresabonnement für 110,- € gegen Nachweis und Bankeinzug. Zusätzlich fallen Versandkosten an: im Inland 41,80€, in Europa 53,40€ und weltweit $87,90 €$. 